\title{
Transpedal lymphatic embolization for lymphorrhea at the graft harvest site after coronary artery bypass grafting
}

\author{
Jung Guen Cha ${ }^{1}$, Sang Yub Lee ${ }^{2}$, Jihoon Hong ${ }^{1}$, Hun Kyu Ryeom ${ }^{2}$, Gab Chul Kim ${ }^{2}$, Young Woo Do ${ }^{3}$ \\ ${ }^{1}$ Department of Radiology, Kyungpook National University Hospital, Daegu, Korea \\ ${ }^{2}$ Department of Radiology, School of Medicine, Kyungpook National University, Daegu, Korea \\ ${ }^{3}$ Department of Thoracic and Cardiovascular Surgery, School of Medicine, Kyungpook National University, Daegu, Korea
}

Received: April 26, 2020

Revised: June 22, 2020

Accepted: June 23, 2020

Corresponding author:

Sang Yub Lee

Department of Radiology, School of

Medicine, Kyungpook National

University, 130 Dongdeok-ro, Jung-

gu, Daegu 41944, Korea

Tel: +82-53-200-5390

Fax: +82-53-422-2677

E-mail: Isyrad@gmail.com

\begin{abstract}
Lymphorrhea is a rare but potentially severe complication that occurs after various surgical procedures. Untreated lymphorrhea may lead to wound dehiscence, infection, and prolonged hospital stay. Currently, there is no standard effective treatment. Early management usually includes leg elevation, drainage, and pressure dressing. However, these methods are associated with prolonged recovery and high recurrence rates. We report a case of lymphorrhea from a calf wound after endoscopic great saphenous vein (GSV) harvesting for coronary artery bypass grafting (CABG). The patient presented with intractable oozing from the postoperative wound on the right calf. Lymphorrhea persisted for 6 weeks despite negative-pressure wound therapy with a long-acting somatostatin. We performed unilateral pedal lymphangiography that confirmed wound lymphorrhea, followed by glue embolization. No recurrence was observed after 8 months of follow-up. This case report demonstrates the successful use of lymphangiography with glue embolization in the control of lymphorrhea after GSV harvesting for CABG.
\end{abstract}

Keywords: Lymphangiography; Lymphatic system; Therapeutic embolization; Therapeutics

\section{Introduction}

Lymphorrhea is a lymphatic leakage from wounds resulting from trauma of the lymphatic vessels; $10 \%$ to $16 \%$ of patients develop lymphorrhea after vein graft harvesting for arterial bypass surgery [1]. Anatomically, large lymphatic vessels lie adjacent to the great saphenous vein (GSV) and are prone to injury during GSV harvesting [2].

When lymphorrhea is persistent and intractable despite conservative treatment including low-fat diet with medium-chain triglycerides, total parenteral nutrition, drainage, somatostatin, and negative-pressure wound therapy (NPWT), it can cause wound dehiscence, infection, and consequently prolonged hospital stay.

Here, we report a purely percutaneous approach for the treatment of persistent and intractable lymphorrhea using unilateral pedal lymphangiography and subsequent $\mathrm{N}$-butyl cyanoacrylate (NBCA) embolization of the injured lymphatic vessel, which was found to be effective.

\section{Case}

This study was approved by the Institutional Review Board of Kyungpook National University Hospital (IRB No. 2020-05078). The patient provided written informed consent for publication of clinical details and images.

A 63-year-old male patient underwent endoscopic GSV harvesting for coronary artery bypass grafting $(\mathrm{CABG})$ due to ischemic heart disease. He had a history of hypertension, diabetes mellitus, and chronic kidney disease. After GSV harvesting, the patient developed wound oozing on the medial side of the right

Copyright (C) 2021 Yeungnam University College of Medicine

This is an Open Access article distributed under the terms of the Creative Commons Attribution Non-Commercial License (http://creativecommons.org/licenses/by-nc/4.0/) which permits unrestricted non-commercial use, distribution, and reproduction in any medium, provided the original work is properly cited. 
calf. This was managed conservatively with NPWT. During the subsequent 6 weeks, the oozing continued. Therefore, direct pedal lymphangiography and percutaneous embolization were requested.

The patient was transferred to the interventional radiology suite and placed in the supine position on the operating table. The foot ipsilateral to the leakage site was selected, and $0.5 \mathrm{~mL}$ of methylene blue was injected intradermally into the web spaces of the right foot. After waiting for 10 minutes, the injected methylene blue was released through the medial calf wound, thereby confirming lymphorrhea (Fig. 1).

A transverse cutaneous incision at the foot dorsum and dissec- tion of the surrounding soft tissue were performed to expose a lymphatic vessel with blue staining. Then, the isolated lymphatic vessel was cannulated using a 26-gauge (G) LG needle (Cook, Bloomington, IN, USA). After accessing the lymphatic vessel, 2 to $3 \mathrm{~mL}$ of contrast agent (Lipiodol; Guerbet, Paris, France) was injected at a rate of $0.2-0.4 \mathrm{~mL} / \mathrm{min}$ using an injection pump. Serial fluoroscopic images were obtained until the interrupted lymphatic vessel leaking into the calf wound was identified. Then, we performed embolization of the culprit lymphatic vessel through initially cannulated 26-G LG needle at the foot dorsum using NBCA and lipiodol mixture in a ratio of 1:4 (Fig. 2). To prevent recurrence, after additional cannulation of the midportion of the culprit
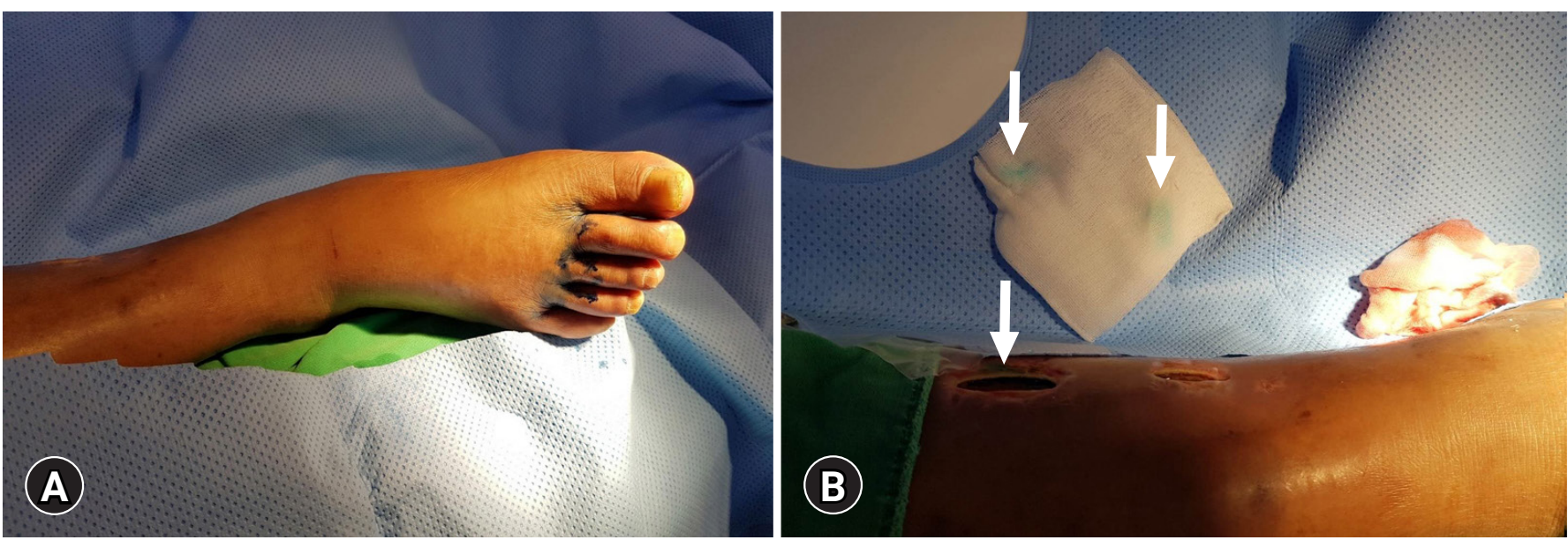

Fig. 1. Photographs of pedal lymphangiography. (A) Photograph of the foot during the subcutaneous injection of methylene blue into the web space. (B) Photograph of the medial calf shows injected methylene blue seeping through the wound (arrows), confirming lymphorrhea.
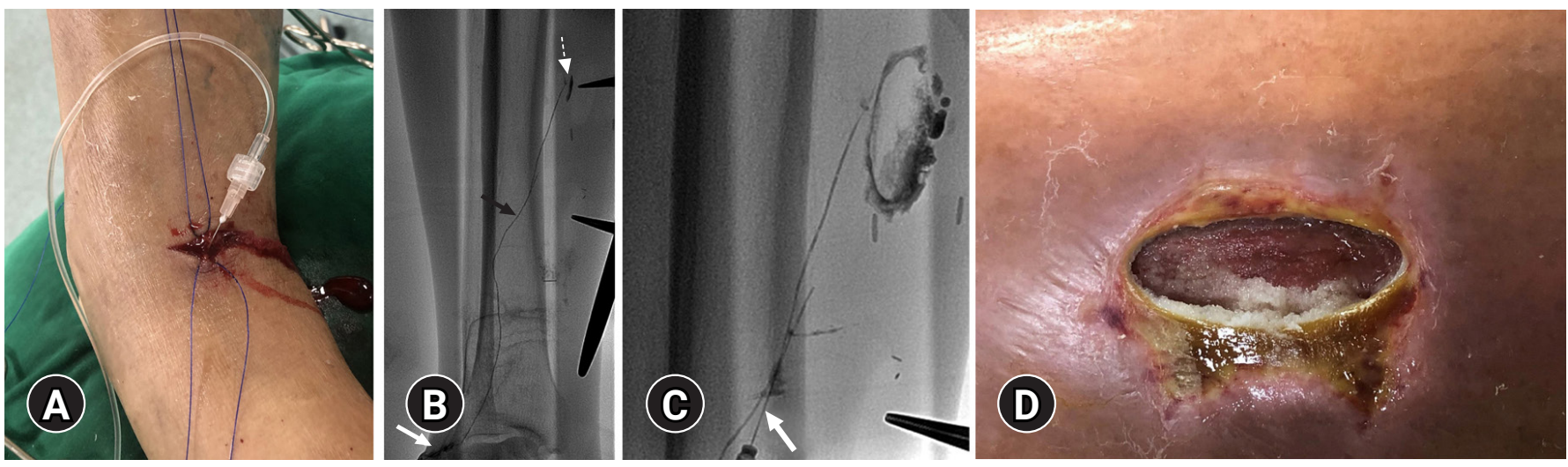

Fig. 2. Percutaneous lymphatic vessel embolization. (A) Photograph of the lymphatic vessel puncture site and transverse incision on the right foot dorsum. (B) Spot radiograph of the calf shows puncture needle (white arrow), normal ascending lymphatic vessel (black arrow), and lipiodol leakage into the calf wound (dashed arrow). (C) Spot radiograph reveals embolized culprit lymphatic vessel using N-butyl cyanoacrylate and lipiodol mixture (mixture ratio, 1:4). Note that additional lymphatic vessel is accessed with needle (white arrow), and additional lymphatic embolization is performed to prevent recurrence. (D) Photograph of the calf wound reveals polymerized glue cast released through the wound. 
lymphatic vessel, further embolization was performed using the same NBCA and lipiodol mixture (Fig. 2C). During percutaneous NBCA embolization, polymerized glue cast was released through the wounds (Fig. 2D). After embolization, initially cannulated proximal lymphatic vessels (Fig. $2 \mathrm{~A}$ ) at the transverse incision site of the foot dorsum were ligated. After successful embolization, the oozing finally stopped. However, despite embolization and wound management for 2 months, the patient underwent skin grafting for prolonged wound exposure and wound dehiscence. Then, the patient was discharged from the hospital.

\section{Discussion}

We described an approach for the management of refractory lymphorrhea using pedal lymphangiography and subsequent NBCA embolization. As in our case, conservative management is recommended before invasive treatment. Conservative options include low-fat diet with medium-chain triglycerides, total parenteral nutrition, drainage, somatostatin, and NPWT. However, lymphorrhea involves a large amount of output and difficult-to-find leakage site and can be refractory to conservative treatment. In such cases, surgical or interventional management has been the standard of care. However, several patients with lymphorrhea are not eligible for surgery because of various reasons. Furthermore, the surgical approach requires imaging workup to ascertain the exact location of the leak. Compared with surgery, lymphangiography with or without embolization can be performed as a diagnostic and therapeutic test. Moreover, lymphangiography is superior to surgery in terms of minimal invasiveness.

Currently, pedal lymphangiography has been replaced with the less cumbersome and more feasible intranodal lymphangiography. The intranodal approach has recently been introduced and remarkably reduced time and effort compared with the conventional pedal lymphangiography approach [3-5]. However, intranodal lymphangiography cannot be performed at the below-theknee level because the lowest lymph node is present in the popliteal area [6].

In conventional pedal lymphangiography, the web space is usually recognized as the standard injection site. However, this approach does not always allow visualization of complete lower leg lymphatics. Shinaoka et al. [6] reported that injection into this web space demonstrates lymphatic vessels only in the anteromedial group. The anteromedial and posteromedial groups did overlap in the leg; however, the anterolateral and posterolateral groups were independent. Therefore, with respect to the region, the site of methylene blue injection should be modified. In our case, dam- aged lymphatic channels were on the anteromedial side. Thus, injecting it into the web space was sufficient to reveal lymphorrhea.

Lymphatic vessel cannulation with a fine needle is challenging. Usually, the lymphatic vessel diameter is smaller than that of a conventional fine needle $(26 \mathrm{G})$. To increase the accuracy of the puncture, parallel advancement of the needle along the lymphatic vessel is important. In this method, the needle tip can cannulate the lymphatic vessel wall multiple times, and slow injection of contrast materials can enable accurate puncture.

In summary, we presented a case of successful percutaneous lymphatic vessel embolization with NBCA for intractable lymphorrhea at the graft harvest site after CABG. Understanding the lymphatic anatomy and relevant techniques is essential for this type of procedure.

\section{Acknowledgments}

\section{Conflicts of interest}

No potential conflict of interest relevant to this article was reported.

\section{Funding}

This research was supported by the Basic Science Research Program through the National Research Foundation of Korea (NRF) funded by the Ministry of Education, Science and Technology (NRF- 2017R1C1B5075931).

\section{Author contributions}

Conceptualization, Investigation: all authors; Formal analysis: JGC, SYL, JH; Methodology: JGC, SYL, HKR, GCK; Resources: JH, HKR, YWD; Supervision: SYL; Writing-original draft: JGC; Data curation, Writing-review \& editing: JGC, SYL.

\section{ORCID}

Jung Guen Cha, https://orcid.org/0000-0002-2519-2120 Sang Yub Lee, https://orcid.org/0000-0001-8529-8229 Jihoon Hong, https://orcid.org/0000-0003-3389-244X Hun Kyu Ryeom, https://orcid.org/0000-0003-4327-8777 Gab Chul Kim, https://orcid.org/0000-0001-7963-7538 Young Woo Do, https://orcid.org/0000-0001-9725-9163

\section{References}

\footnotetext{
1. Unno N, Yamamoto N, Suzuki M, Tanaka H, Mano Y, Sano M, et al. Intraoperative lymph mapping with preoperative vein mapping to prevent postoperative lymphorrhea following paramalleolar bypass surgery in patients with critical limb ischemia.
} 
Surg Today 2014;44:436-42.

2. Heitink MV, Schurink GWH, de Pont CDJM, van Kroonenburgh MJPG, Veraart JCJM. Lymphedema after greater saphenous vein surgery. Eur J Vasc Endovasc Surg 2009;38:656.

3. Kos S, Haueisen H, Lachmund U, Roeren T. Lymphangiography: forgotten tool or rising star in the diagnosis and therapy of postoperative lymphatic vessel leakage. Cardiovasc Intervent Radiol 2007;30:968-73.

4. Rajebi MR, Chaudry G, Padua HM, Dillon B, Yilmaz S, Arnold RW, et al. Intranodal lymphangiography: feasibility and prelimi- nary experience in children. J Vasc Interv Radiol 2011;22: 1300-5.

5. Nadolski GJ, Itkin M. Feasibility of ultrasound-guided intranodal lymphangiogram for thoracic duct embolization. J Vasc Interv Radiol 2012;23:613-6.

6. Shinaoka A, Koshimune S, Suami H, Yamada K, Kumagishi K, Boyages J, et al. Lower-limb lymphatic drainage pathways and lymph nodes: a CT lymphangiography cadaver study. Radiology 2020;294:223-9. 